Stephen G. Hickey*

\title{
The Photoelectrochemistry of Assemblies of Semiconductor Nanoparticles at Interfaces
}

https://doi.org/10.1515/zpch-2018-1178

Received March 14, 2018; accepted March 24, 2018

Abstract: The application of photoelectrochemical methods presents the researcher with a powerful set of versatile tools by which photoactive materials, such as semiconductor quantum dots, at conductive interfaces may be interrogated. While the range of photoelectrochemical techniques available is quite large, it is surprising that very few have found their way into common usage within the nanoparticle community. Here a number of photoelectrochemical techniques and the principles upon which they are based are introduced. A short discussion on the criticality of ensuring the nanoparticles are reliably anchored to the substrate is followed by an introduction to the basic set of equipment required in order to enable the investigator to undertake such experiments. Subsequently the four techniques of transient photocurrent response to square wave illumination, photocurrent spectroscopy, intensity modulated photocurrent spectroscopy and intensity modulated photovoltage spectroscopy are introduced. Finally, the information that can be acquired using such techniques is provided with emphasis being placed on a number of case studies exemplifying the application of photoelectrochemical techniques to nanoparticles at interfaces, in particular optically transparent electrodes.

Keywords: charge injection; EMAS; IMPS; nanoparticles; optical bleaching; optical properties; photocurrent transients; photoelectrochemistry.

\section{Introduction}

Photoelectrochemistry is by its very nature an interdisciplinary subject that brings together different aspects of chemistry, solid-state physics and electronics. This has resulted in the development of a remarkably wide range of techniques that can be brought to bear in order to characterise photoelectrochemical

\footnotetext{
*Corresponding author: Stephen G. Hickey, School of Chemistry and Biosciences, University of Bradford, Bradford, West Yorkshire BD7 1DP, UK, Tel.: +44 (0) 1274235527 , e-mail:S.Hickey1@bradford.ac.uk
} 
systems. Photoelectrochemistry, primarily due to the diverse range of techniques that it encompasses, has been instrumental in providing access to a wealth of information concerning the electronic makeup of a wide assortment of materials. In earlier studies optoelectrochemical techniques have been employed to study systems as varied as $\mathrm{WSe}_{2}[1,2], \mathrm{MoSe}_{2}$ [1], p- and n-InP [3], PtS 2 [4] and CdSe [5] in solutions of $\mathrm{KI}$ and $\mathrm{KCl}$. Photoelectrochemical techniques have also been employed to interrogate processes at dye sensitised solar cells $[6,7]$ where the role that the nanocrystalline metal oxide plays in charge transfer is of paramount interest. Given the fact that semiconductor nanoparticle (SCNP) derivatised interfaces have the potential to impact many important technology spheres especially those that depend on the movement of charge and its subsequent phase transfer such as photodetectors [8, 9], light emitting diodes [10], solid state lighting [11, 12], solar cells [13, 14], batteries [15], etc. it is surprising that photoelectrochemical techniques have not received wider penetration and acceptance within the field of nanoparticle (NP) characterisation particularly with respect to the evaluation of device performance.

One of the most important aspects of photoelectrochemical techniques is that they measure the response of the device as a whole and hence the combined role of all of the conductive pathways and accompanying interfacial charge transfers. While this is a significant advantage if one wishes to evaluate the performance of the over-all device it poses a number of challenges if one is interested in the performance of say one interface as is usually the case in the laboratory. One area where this is of major significance in nanoparticle systems is the nanoparticle/substrate interface where often a bifunctional molecule is used to tether the nanoparticles to the underlying substrate. In many cases the substrate is composed of glass coated with a transparent conductive coating, e.g. doped tin or indium oxide, which plays the role of an optically transparent electrode (OTE). This OTE is the back contact where the electron may transfer from the nanoparticle (oxidative/anodic current) and enter the circuit, the electron vacancy (hole) that remains being filled by a solution species. Alternatively, the electron may transfer from the OTE to the nanoparticle and subsequently to a solution species (reductive/cathodic current). In all of this the bifunctional linking molecule is far from benign given its role as an in-series circuit component through which the charge carriers must transit. The main functional groups employed in the linking of nanoparticles to the electrode interface are the thiols, carboxylic acids, amines and trimethoxysilanes. In one such study [16], in which a series of important short chained, bifunctional linker molecules was studied, it was found that the mode of linking was not as expected for a number of the smaller molecules under study. The conductive substrates were treated with six different linking molecules [(i) oxalic acid (OA), (ii) malonic acid (MA), (iii) thioacetic acid (TAA), 
(iv) mercaptopropionic acid (MPA), (v) thioglycolic acid (TGA) and (vi) hexandithiol (HDT)], i.e. two diacids, three acid thiols and a dithiol, respectively (see Figure 1). Of the six molecules only half could be said to have behaved in a reasonably predictable manner. Molecule (i) was too acidic and etched the oxidic interface and (ii), although small and therefore a desirable candidate with respect to its potentially low resistance, gave photocurrents that were approximately two orders of magnitude lower than the dithiol. The photocurrents received using TAA as the linker were opposite in sign to all other measured photocurrents. The change in sign of the current signifies that the net current generated using this electrode is flowing in the opposite direction compared to all of the other electrodes under investigation in this study. As the relative position of the nanoparticulate $\mathrm{PbS}$ conduction band is not expected to alter with respect to the substrate energy levels, the change in the direction of the current may be due to a chemical modification of the PbS. The MPA, TGA and HDT all provided a reasonable response in the photocurrent transients with current densities of between 20 and $30 \mu \mathrm{A} \mathrm{cm}^{-2}$ being recorded for this system.

Another challenge that one faces when attempting to link nanoparticles to surfaces is not only that the bifunctional linker attachment mode is correctly orientated but also that the surface coverage of the linker is sufficiently high so as to allow the deposition of meaningful quantities of the photoactive material at the interface. This issue was addressed by a re-examination of the standard protocol [17]. The general procedure for the attachment of nanoparticles to an OTE is to immerse the OTE in a solution of a trimethylsilane, such as mercaptpropyltrimethylsilane (MPTMS) or aminopropyltrimethylsilane (APTMS), subsequent to the functionalisation of the surface with hydroxyl groups by immersion in highly basic media, usually 3-5 M potassium hydroxide. This procedure has a number of complications associated with it amongst which are: the conductive oxide layer

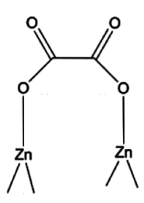

a

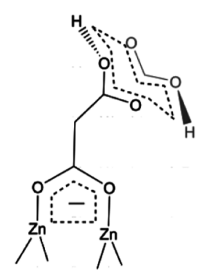

b

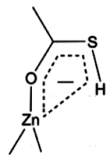

C

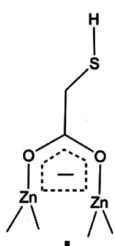

d

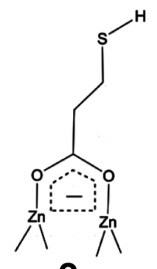

e

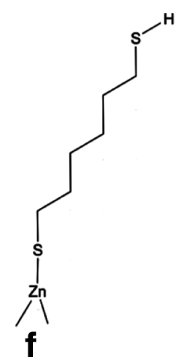

Fig. 1: Schematic of the most probable linker bonding modes to the $\mathrm{ZnO}$ substrate: (a) OA; (b) MA, (c) TAA; (d) TGA; (e) MPA; and (f) HDT. (Reprinted with permission from Ref. [16]. Copyright 2013 American Chemical Society.) 
undergoes etching in strong basic media; the presence of water, during the trimethylsilane treatment, either as a residue after treatment with the base or present in the atmosphere, causes the trimethylsilane to polymerise; the correct alignment of the three methoxy groups of the trimethylsilane with the hydroxyl groups at the surface of the OTE is not guaranteed and often results in an unpredictable surface density of the linking molecules; and crucially the deposition of many types of nanoparticles relies on the NP surface, often coated with an organic ligand, being somehow available to the pendant trimethylsilane group providing the attachment. However much of this uncontrollability can be mediated if, at first, the linking molecule (MPTMS or APTMS) is substituted for the initial surface bound organic ligand by attaching the thiol or amine to the NP surface followed by deposition onto the OTE from solution. Using this new protocol a wide variety of semiconductor and metallic nanoparticles could be attached to a range of important oxide materials ( $\mathrm{TiO}_{2}, \mathrm{ZnO}_{2}, \mathrm{SiO}_{2}$, etc.). To achieve this, the NPs first underwent ligand exchange for the trimethylsilane. For a typical ligand exchange, MPTMS was added to a solution of the NPs in dry toluene. This solution was the shaken for a few seconds and the particles subsequently precipitated and separated by centrifugation. Excess ligands were removed by washing a second time and the particles were redispersed in dry dichloromethane. When the surface concentration of nanoparticles using this method was compared with the standard method applied to two different molecules (MPTMS and MPA) using cyclic voltammetry, fractional coverages of $>80 \%$ were recorded (c.f. $14 \%$ using the standard protocol) while the time consuming and intricate step of functionalising the substrates before deposition had been avoided. Significantly, the optical properties of absorption and emission for the substrate nanomaterials were found to be essentially unchanged from that of the solution phase materials (see Figure 2).

\section{The basic photoelectrochemical measurement requirements}

As a general, working definition photoelectrochemistry can be thought to be concerned with "the electrochemical behaviour of interfaces consisting of a photoactive electrode, which is electronically excited through irradiation with light, in the presence of an appropriate electrolyte" [18]. This clearly suggests that the basic components of such a system are a potentiostat to apply/measure the potential at the interface and a light source to effect optical excitation. With respect to their wavelength output, the light sources used may be broadband (bulb) or monochromatic (e.g. laser or monochromated source). In the case of 

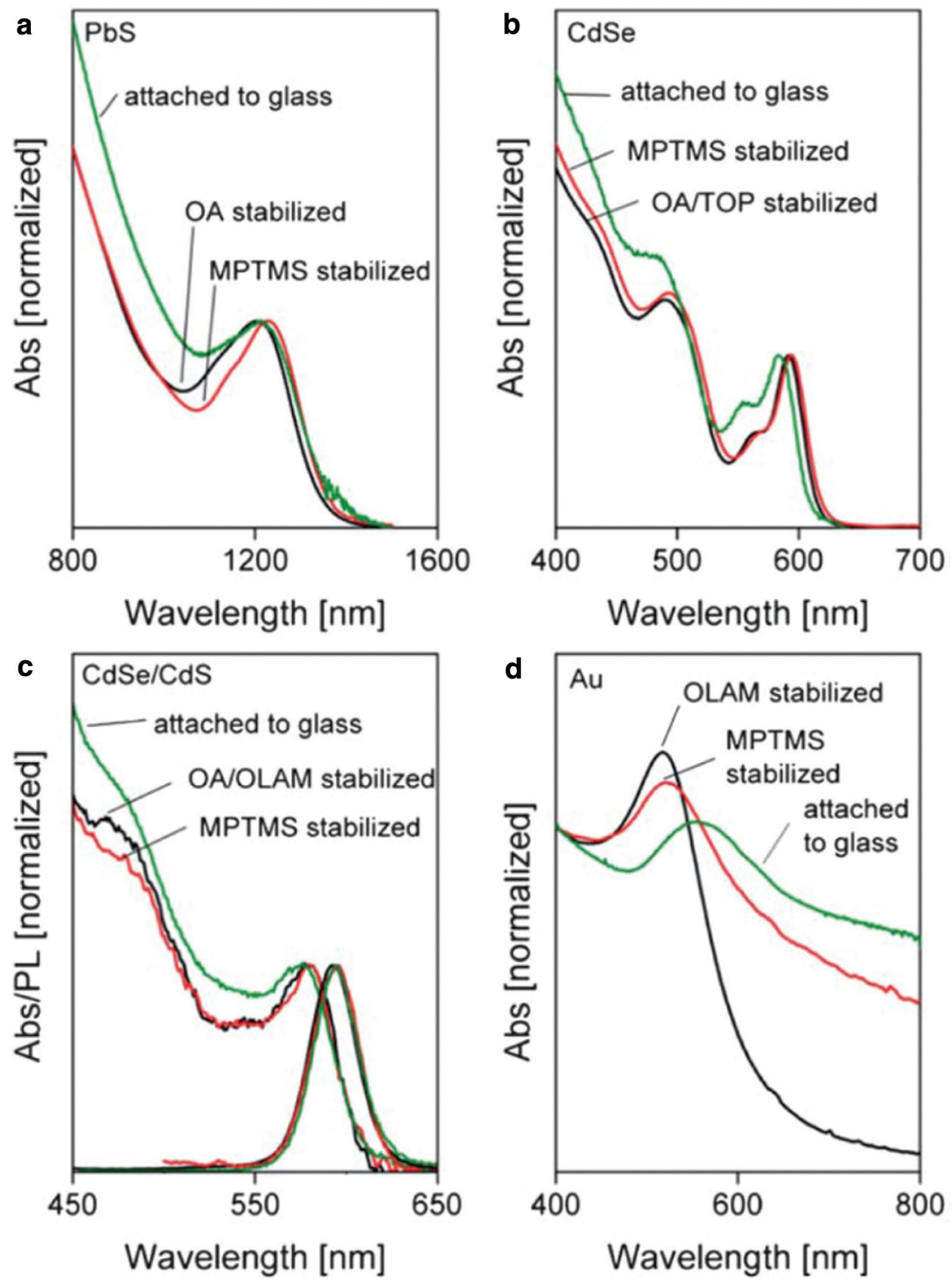

Fig. 2: Comparison of the absorption spectra of (a) PbS, (b) CdSe, (c) CdSe/CdS core-shell and (d) Au NCs stabilised (i) with their initial ligands, (ii) with MPTMS and (iii) after attachment to a glass substrate. The spectra of the semiconductor NCs have been normalised to the $1 \mathrm{~S}_{\mathrm{e}}-1 \mathrm{~S}_{\mathrm{h}}$ transition whereas the spectra of the Au NCs have been normalised to their extinction value at $400 \mathrm{~nm}$. (Reproduced from Ref. [17] with permission from The Royal Society of Chemistry.) 


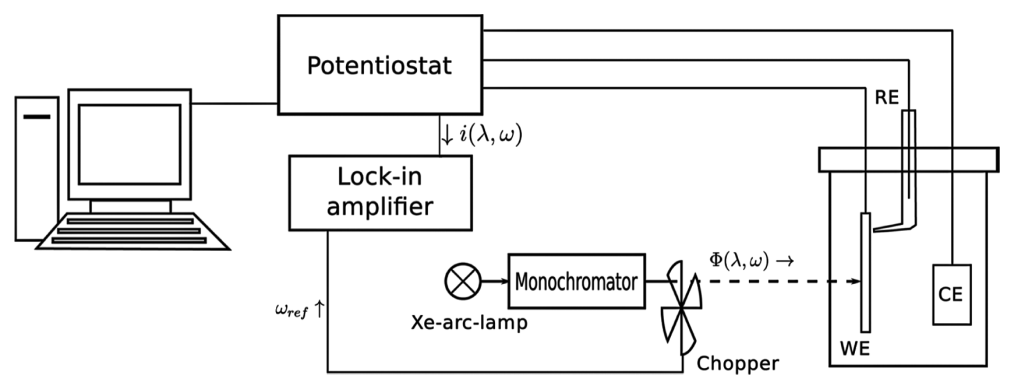

Fig. 3: Basic experimental setup required for photocurrent spectroscopy. The monochromator may be omitted at the sacrifice of wavelength dependent studies. (Reprinted with permission from Ref. [18]. Copyright 2014 American Chemical Society.)

the response signal being of sufficient intensity so as to be directly recorded this arrangement is sufficient, but in many cases a lock-in amplifier is included in the experimental set-up as the signal to noise ratio may be quite low, in fact the response signal may be imperceivable using dc methods. The role of the lock-in amplifier is to act as a phase sensitive detector (adjustable bandwidth filter) singling out only those signals that occur at the particular frequency of interest which are then filtered out from the rest of the signal. This allows for a dynamic reserve (detection ability) in modern instruments of up to $120 \mathrm{~dB}$, which translates as an ability to extract $1 \mu \mathrm{V}$ of signal in amongst $1 \mathrm{~V}$ of noise. The signal at the frequency of interest will be contained in amongst the return signal and may be generated in a number of ways, of which the following are the two most common: (i) application of a modulated electrically generated signal to the electrode interface or (ii) application of a modulated optical signal to the OTE. In both cases it is expected that the application of the modulated probe signal will be accompanied by a return signal which is similarly modulated and which may or may not be accompanied by a phase difference, depending on the response times of the system and the frequency of the probe modulation. Where the light intensity is modulated, the modulation may be supplied either by a variable voltage applied directly to the light source, e.g. a modulated voltage applied to an LED, or by using a chopper. Using just the basic set-up described here (see also Figure 3) many optoelectrochemical techniques are easily implemented and below are listed just a few. The interested reader is referred to the literature if they should require details $[18,19]$.

Before one embarks on any optoelectrochemical investigations it is always worth to carry out linear sweep voltammetry (LSV) or cyclic voltammetry (CV) beforehand. These techniques will inform the investigator as to the range of applied potentials across which the interface is electrochemically stable. 
Additionally, from CV it is possible to deduce which redox peaks are coupled and in some circumstances information concerning the species undergoing electrochemistry may be discerned. The effects of operating outside of the region of electrochemical stability have been reported on for CdSe nanoparticles on an OTE where it was found that the optical properties of the material, specifically the photoluminescence, were adversely affected [20]. Upon scanning the applied potential of the OTE in the negative direction, from $0 \mathrm{~V}$ to $-2.0 \mathrm{~V}$ using a Pt-wire as a pseudoreference, it was reported that the electrode had lost almost $50 \%$ of its initial luminescence intensity at $-2.0 \mathrm{~V}$ but recovered to approximately $80 \%$ of the initial value upon returning to $0 \mathrm{~V}$. In contrast, when the potential was scanned in the positive direction form $0 \mathrm{~V}$ to $+2.0 \mathrm{~V}$, the electrode lost more than $35 \%$ of its initial intensity which it failed to regain when the applied potential returned to the $0 \mathrm{~V}$ position. Moreover, if it is possible to undertake electrochemical impedance spectroscopy (EIS) it is highly recommended to do so. EIS is capable of providing a wealth of information, much of which is complementary to photoelectrochemical methods. Indeed there are a few other methods that provide such numerous insights into reaction mechanisms, kinetics, topology and other physicochemical quantities with such a minimum of effort.

\subsection{Transient photocurrent response to square wave illumination}

In this technique the photoactive electrode interface is excited using square wave illumination and the photocurrent response of the system is recorded as a function of time and/or applied potential. Evaluation of the transient profiles provides insights into the rates of the different charge transfer processes involved, their competition and the dominant direction of the charge carrier flux. It is insightful to undertake this experiment as a function of the illumination intensity so as to ensure that the optoelectrochemical response of the electrode remains linear across the range of illumination intensities and applied potentials over which the experiment is being undertaken.

\subsection{Photocurrent spectroscopy}

Here the photocurrent generated by the photoactive electrode is recorded as a function of the wavelength of the incident light (this technique assumes the incorporation of a monochromated light source or the like.). This technique is analogous to UV-vis spectroscopy, where the difference in intensity due to 
the absorbance of the sample with respect to a reference is recorded. In photocurrent spectroscopy the resulting charge carrier flux generated due to the absorbance of light by the photoactive component(s) is determined. This has the advantage of discriminating against all but the optically active component at the interface. Additionally, with the use of a calibrated photodiode, the all important incident photon to current conversion efficiency (IPCE) can be deduced.

\subsection{Intensity modulated photocurrent spectroscopy (IMPS)}

This is a powerful technique to derive information concerning the kinetics of the charge transfer associated with the photoelectrochemical processes that occur at an illuminated electrode. IMPS experiments are carried out in the frequency domain. A photoelectrochemically active electrode is excited under potentiostatic control using a continuous light intensity onto which a small amplitude, sinusoidally modulated light intensity is superimposed. The corresponding ac response of the generated photocurrent is measured for its phase sensitivity by means of a frequency response analyzer (FRA) or a dual-phase lock-in amplifier. The ac light intensity is chosen to be sufficiently small (typically $10 \%$ or less of the dc light intensity) to guarantee a linear response of the system under investigation. A number of common light sources can be employed for such experiments such as LEDs, continuous lasers modulated by acousto-optic devices and diode lasers.

\subsection{Intensity-modulated photovoltage spectroscopy (IMVS)}

IMVS is the galvanostatic analogue of IMPS. As is the case for IMPS, the electrode is perturbed by a small amplitude, sinusoidally modulated light intensity and the modulation swept over a wide range of frequencies. However, whereas for IMPS the frequency-dependent complex photocurrent of the electrode under potentiostatic conditions was measured, in IMVS the electrode is kept under constant current conditions and the modulated voltage response is recorded. While IMVS data can be collected at any constant current value, the measurements are usually made under conditions of zero current. Thus, for probing a solar cell the experiment would be performed under open-circuit conditions. IMVS therefore provides information concerning the electron lifetimes and recombination kinetics at open circuit. 


\section{What can be learned from the photoelectrochemical probing of the SCNP/ OTE interface - some case studies}

As one generally wishes to probe a particular optically active interface, but will receive a signal that reflects the response of the system as a whole, it is best, even necessary, to first evaluate the electrochemical and photoelectrochemical response of the system with all components, except the optically active component of interest, present. This allows the "bare" system to be evaluated and subsequently used as a reference system. Additionally, it is best to build up the working electrode (i.e. the interface of interest, here most likely the OTE) in a layer by layer fashion, evaluating all changes that occur at the electrode as each layer is sequentially added, before the addition of the nanoparticles. In many of the initial photoelectrochemical studies of nanoparticles at OTE interfaces the nanoparticles were generated with the linker derivatised OTE in-situ, i.e. present in the reaction vessel during the NP growth. In the work of Riley et al. CdS NPs were deposited onto the MPTMS derivatised tin oxide during the nanoparticle synthesis from THF solutions of cadmium perchlorate and hydrogen sulfide in the presence of hexane dithiol (HDT) as ligand [21-24]. Peter and co-workers [25] extended this procedure to the deposition of CdS nanoparticles onto $\mathrm{TiO}_{2}$ substrates by first synthesising the CdS NPs in the presence of mercaptopropionic acid in place of HDT and subsequent deposition onto the $\mathrm{TiO}_{2}$ coated electrode. The former works were concerned with the deposition of CdS NPs of different average diameters, their photoelectrochemical response and subsequent fitting of the data to specific models of the charge carrier transfer so as to account for the photocurrent profiles observed. Initially the model considered was a three level model (nanoparticle valence and conduction bands plus the fermi level of the OTE) and the high frequency domain data could be fit quite well using this basic interpretation. However, later it was deemed necessary to account for the photocurrent response at lower frequencies, where the data points were observed to curve back in towards the origin rather than condense to the real axis at right angles, by introducing a fourth level, that of surface states. As is usually the case, the picture of what processes were in operation at the interface was built up using a number of photoelectrochemical techniques: photocurrent transients and steady-state photocurrents recorded at different applied potentials, potential modulated absorption spectroscopy (EMAS) across a range of modulation amplitudes as well as IMPS. The photocurrent transients allowed the identification of both the presence and the energy level position of the surface states while the steady-state photocurrents allowed the energy position of the conduction level to be identified. In addition to the information 
gained from the photocurrent transients and steady state values EMAS, where a modulated potential is applied to the OTE/NP interface resulting in the filling of interband energy levels at the nanoparticle and effectively closing them down as charge carrier conduction channels or, at potentials where the conduction band states are being addressed, denying electrons that have undergone photonic excitation access to the now filled levels, was also employed. This resulted in a bleaching of the absorbance profile in the parts of the spectrum where the nanoparticle absorbance was attenuated. This allowed an answer to the question of what is the cause of the bleaching of the absorbance: filling of the conduction band levels or changes due to the presence of surface states, to be proposed. Because changes in the absorbance profile were not observed until the substrate Fermi level was coincident with the conduction band edge of the CdS nanoparticles clearly supports a band-filling model of the bleaching process and indicated surface states were not involved in the process. After consideration of the IMPS data and in light of the information gleamed from the suite of techniques used it could be demonstrated that four steps must be considered in the charge separation process of the nanoparticles at the OTE interface; electron tunnelling from the nanoparticle conduction band to the Fermi level of the substrate $\left(\mathrm{k}_{\mathrm{e}}\right)$, hole capture by the redox species $\left(\mathrm{k}_{\mathrm{h}}\right)$, conduction band to valence band recombination at the nanoparticle $\left(\mathrm{k}_{\mathrm{r}}\right)$ and back electron transfer from the substrate to the surface states on the NP $\left(k_{b}\right)$. Figure 4 outlines schematically the aforementioned processes and where they occur in such systems with respect to the nanoparticle/substrate interface. Detailed analysis of the IMPS data allowed $\mathrm{k}_{\mathrm{b}}, \mathrm{k}_{\mathrm{r}}$ and $\mathrm{k}_{\mathrm{h}}$ to be determined and information on the dependence of $\mathrm{k}_{\mathrm{e}}$ on applied potential could also be inferred. It was
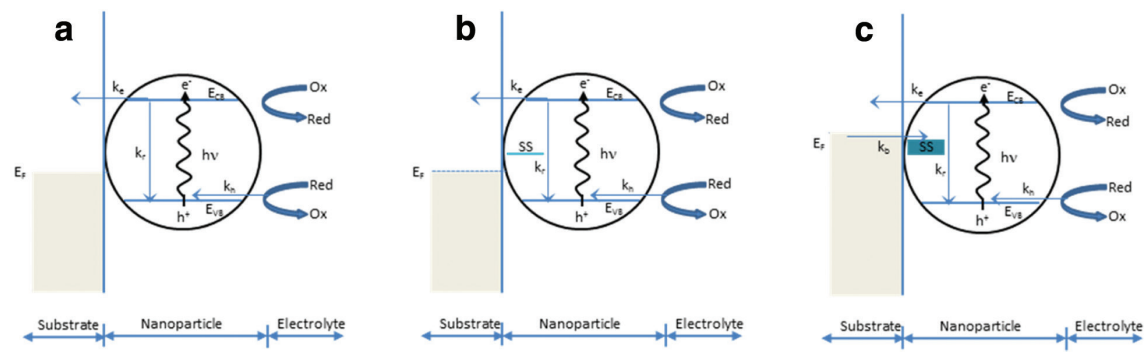

Fig. 4: Schematic depicting (a) the three level model, (b) the four level model with the electrode Fermi level $\left(E_{F}\right)$ below the surface state energy level and $(c)$ the four level model with the electrode Fermi level $\left(E_{F}\right)$ above the surface state energy level. The electrode processes of interest are: the rate of electron transfer of electrons from the nanoparticle conduction band to the substrate $\left(k_{e}\right)$, the rate of charge carrier (exciton) recombination at the nanoparticle $\left(k_{r}\right)$ and the rate of electron transfer of electrons from the substrate to the surface states on the nanoparticle $\left(k_{b}\right)$. The latter is only of significance once $E_{F}$ is higher in energy than the surface state level. 
also found that $\mathrm{k}_{\mathrm{b}}$ and $\mathrm{k}_{\mathrm{e}}$ were observed to be potential dependent, whilst $\mathrm{k}_{\mathrm{r}}$ and $\mathrm{k}_{\mathrm{h}}$ were potential independent for the system studied. Additionally, consideration of the potential dependence of the rate constants allowed the energies of the conduction band and hole traps to be determined.

In a study based along similar lines, Vanmaekelbergh and co-workers [26, 27] used photoelectrochemistry to probe metal sulfide nanoparticles adhered to a metal (gold) electrode. In a study of polyphosphate coated CdS nanoparticles on self-assembled monolayers (SAM) of hexanedithiol and nonanedithiol, they were able to directly determine the nanoparticle related tunnelling rates therefore providing information concerning the tunnelling sequence. By varying the temperature, they were able to distinguish between tunnelling and electrochemical processes. Additionally, while the tunnelling rates perceived were rather low, it was observed that tunnelling could still compete with excited-state relaxation within the particle, primarily due to the long-lived excited state in the lead salts. Due to the low tunnel rates and the weak electronic coupling they were also able to infer that the particles are not bound to the surface by a sulfur-gold interaction but by the stabilising polyphosphate chains. In a further study [28] they were able to determine the distance dependence of the tunnelling currents using a series of three bisulfide spacer molecules [oligo(cyclohexylidenes)]. Using IMPS they were able to report on the electron tunnelling rates for both the conduction band to substrate and trap state to substrate transfers and to reveal that the tunnelling dependence of both were linear on a semi-logarithmic plot of $\log (\mathrm{k})$ versus the separation distance of the CdSe nanoparticles from the substrate.

In a recent in-depth EMAS study of CdSe nanoparticles on FTO coated glass [29] not only was the dependence of the optoelectronic properties on the NP size determined but so too was the effect of the nanoparticle geometry. A distinct advantage that this study had over many of the earlier ones is that the quality of the nanoparticulate material, with respect to its polydispersity, was distinctly improved. This allows for the identification and inspection of quite a number of optical transitions to be undertaken, for the material in this investigation transitions between the $1 S_{3 / 2}-1 S_{e}, 2 S_{3 / 2}-1 S_{e}, 1 S_{1 / 2}-1 S_{e}$ and $2 S_{1 / 2}-1 S_{e}$ levels (see Figure 5). As the changes in absorbance were observed to kick in at an applied potential of $-1.05 \mathrm{~V}$ versus the silver/silver chloride this may then be taken as the energetic position of the conduction band. An additional band, not observed in the absorbance profile, is observed in the photochemical data. As the onset point of this band is identical to all of the other bleached transitions it would appear that this additional signal is directly connected to the population of the $1 S_{e}$ state. Therefore, it can be assumed that this band, which appears at $550 \mathrm{~nm}$, corresponds to an absorption of the CdSe particles in their exited states. This additional feature may result from Coulombic interactions of the injected charge with the optically 

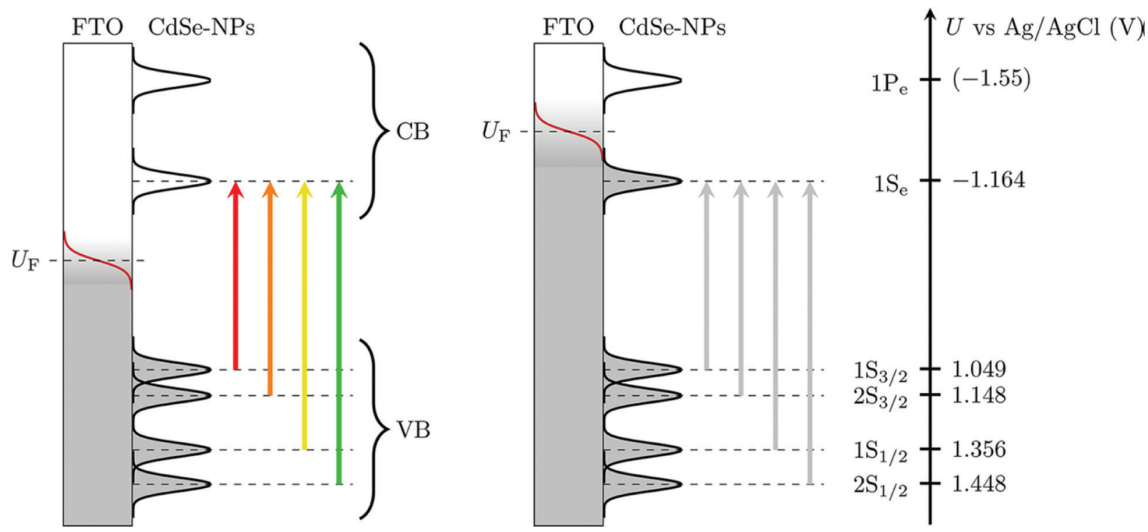

Fig. 5: Schematic of the transitions bleached via the population of the conduction band $1 \mathrm{~S}_{\mathrm{e}}$ state and the calculated potential positions of the individual electronic states within the bands versus the $\mathrm{Ag} / \mathrm{AgCl}$ reference electrode. The valence band states have been calculated on the basis of the potential determined for the $1 \mathrm{~S}_{\mathrm{e}}$ state according to Eq. 2 . The potential of the $1 \mathrm{P}_{\mathrm{e}}$ state is estimated on the basis of results published by Guyot-Sionnest and co-workers [30]. $U_{\mathrm{F}}$ denotes the Fermi potential (corresponding to the Fermi energy). (Reprinted with permission from Ref. [29]. Copyright 2017 American Chemical Society.)

excited electron-hole pair. This would in turn lead to changes in the selection rules and increase the transition probability of initially forbidden transitions. Because the electrochemical potential of the $1 S_{e}$ level has been determined the potential positions of the electronic states within the upper portion of the valence band can now be found. Since we are dealing with excitonic transitions the exciton binding energy, i.e. the Coulomb interaction between the electron and hole, needs to be considered:

$$
U_{g}=U_{g, o p t}+\frac{1.8 e}{4 \pi \varepsilon_{r}^{\infty} \varepsilon_{0} r}
$$

where $U_{g}$ is the potential difference between the valence and the conduction band, $U_{g, o p t}$ the potential value, which can be determined from optical spectroscopy, $e$ the elementary charge, $\varepsilon_{r}^{\infty}$ the relative permittivity at high frequency, $\varepsilon_{0}$ the permittivity of the vacuum and $r$ is the radius of the spherical particle. The potential of the individual valence band state, $U_{S_{h}}$ with respect to the reference is given by:

$$
U_{S_{h}}=U_{0}+\frac{\Delta E_{o p t}\left(S_{h}-1 \mathrm{~S}_{\mathrm{e}}\right)}{e}+\frac{1.8 e}{4 \pi \varepsilon_{r}^{\infty} \varepsilon_{0} r}
$$

where $U_{0}$ is the electrochemical potential of the $1 \mathrm{~S}_{\mathrm{e}}$ state as determined by EMAS and $\Delta E_{\text {opt }}\left(S_{h}-1 \mathrm{~S}_{\mathrm{e}}\right)$ is the energy of the transition between the respective 
valence band state and the $1 \mathrm{~S}_{\mathrm{e}}$ state, with all other symbols having their usual meanings.

When applied to a range of nanoparticle sizes the EMAS spectra of each sample demonstrated a bleach of all four optical transitions and the appearance of the induced absorption band. However the smaller particles showed lower intensities in general. As that the absorption coefficient is directly proportional to the particle volume, the decrease in signal intensity from the largest size to the smallest size used in the study is expected to be less than $10 \%$. Thus, the lower intensities were attributed to the ability of the smaller particle sizes to attach to the OTE during the deposition procedure being less efficient due to possessing a more strongly curved surface. Once absolute energy value for the conduction band and valence band edges of the different sizes of CdSe nanocrystals had been determined by EMAS the data was compared with the theoretically predicted curves based on the effective mass approximation (EMA) and a tight binding approach (TB) reported by Sapra and Sarma [31]. Whereas the measured data closely matches the size-dependent band edges calculated by the tight binding approach the EMA increasingly overestimates the band gap for decreasing particle size. This is due to the assumption that the charge carriers are confined by an infinite energy barrier.

The application of photoelectrochemical techniques to the investigation of nanoparticles of different dimensionalities is of particular interest as the density of states (DOS) differs significantly as dimensionalities change. For example, in contrast to three dimensional nanoparticulate materials, two dimensional (2-D) nanoparticulate systems possess a 2-D electron gas for which the DOS is constant. Hence the electronic landscape of 2-D systems is such that quasi-continuous electronic bands are present and hence a greater number of conduction band states which can be addressed. Also of interest is that while spherical nanoparticles are inherently geometrically isotropic 2-D materials by their very nature are geometrically anisotropic. This anisotropy coupled with the release of the quantum confinement barrier in one direction makes such investigations particularly attractive. The geometry under investigation here was platelets which were observed to have four distinct optical transitions in their solution phase absorbance profile, one of which was due to an additional fraction of platelets which had not been fully removed. However, three distinct peaks could be discerned in the difference spectra and, as in the case of the spherical nanoparticles, all peaks could be observed to bleach and all three had the same bleach potential onset. There was however a significant difference in the EMAS signature of the platelets in that a large discrepancy in the three inflection point positions was observed. As the inflection point is interpreted to be the band edge potential of the lowest sub-band of the conduction band it is expected to be the same value in all three 
cases. It is clear that this discrepancy requires further investigation so as to correctly assign its significance.

\section{Conclusion}

Photoelectrochemistry techniques provide a versatile platform from which a range of important electronic properties can be interrogated. They range from the most basic measurements such as photocurrent transients (intermittent illumination of the electrode interface while recording the rise and fall of the photocurrent profile as a function of time) to slightly more elaborate set-ups such as EMAS (applying a modulated potential at the electrode interface while recording any changes in the absorbance). While it was not possible to provide an exhaustive survey of all possible methods, the experimental probe variables that one is most likely to be interested in modulating are the potential, current and light intensity and the main return signals to record are the photocurrent, photovoltage and/or absorbance. It is by understanding the interrelationship of all of these parameters that the complex energy landscape within materials can be better understood.

If electrochemistry may be considered to be spectroscopy using electrons then its marriage with optical spectroscopy where we use photons is a natural and obvious progression. Using most modern potentiostats allows one to finely tune, often with sub-millivolt precision, the filling of the electronic states within a material and with a monochromatic light source one can probe these changes with sub-nanometre resolution of the photon energy. Such fine degree of control over the experimental parameters applied to the probing of the energetic makeup of materials can be found in few techniques that are presently openly accessible to the experimentalist.

Moreover all of these studies mentioned here demonstrate one of the most powerful aspects of what are essentially electrochemical techniques, i.e. all of the energy levels identified are with respect to a known reference electrode whose potential with respect to the vacuum level can be ascertained. Hence, all potentials measured in these systems can be related to the vacuum level. There can be few, if any techniques that can be implemented under normal laboratory conditions that have the ability to report energy level values with respect to a known absolute energy level position with such ease of assembly and financial outlay as can photoelectrochemical techniques.

Acknowledgements: It gives me great pleasure to dedicate this work to this special issue celebrating the $60^{\text {th }}$ birthday of Alexander Eychmüller. Alex, I look forward 
to the equally great pleasure of having the privilege of contributing to a special issue celebrating your next milestone birthday. Congratulations on your momentous achievements to date and I am proud to have been associated, even if only in some small part, with your grand scheme. Financial support for the much of the work presented here was provided by the Deutsche Forschungsgemeinschaft (DFG) through grants HI-1113/3-1 and HI-1113/5-1 and Science Foundation lreland's ETS Walton Visitor award scheme grant 11/W.1/12085, is greatly acknowledged.

\section{References}

1. S. Prybyla, W. S. Struve, B. A. Parkinson, J. Electrochem. Soc. 1587 (1984) 131.

2. T. Sakata, E. Janata, W. Jaegermann, H. Tributsch, J. Electrochem. Soc. 133 (1986) 339.

3. R. L. Cook, P. F. Dempsey, A. F. Sammells, J. Electrochem. Soc. 133 (1986) 1821.

4. W. Jaegermann, T. Sakata, E. Janata, H. Tributsch, J. Electroanal. Chem. 189 (1985) 65.

5. R. H. Wilson, T. Sakata, T. Kawai, K. Hashimoto, J. Electrochem. Soc. 132 (1985) 1082.

6. G. Franco, J. Gehring, L. M. Peter, E. A. Ponomarev, I. Uhlendorf, J. Phys. Chem. B 103 (1999) 692.

7. L. M. Peter, K. G. U. Wijayantha, Electrochem. Commun. 1 (1999) 576.

8. S. A. McDonald, G. Konstantatos, S. G. Zhang, P. W. Cyr, E. J. D. Klem, L. Levina,

E. H. Sargent, Nat. Mater. 4 (2005) 138.

9. G. Konstantatos, I. Howard, A. Fischer, S. Hoogland, J. Clifford, E. Klem, L. Levina, E. H. Sargent, Nature 442 (2006) 180.

10. N. Pradhan, D. D. Sarma, J. Phys. Chem. Lett. 2 (2011) 2818.

11. S. K. Panda, S. G. Hickey, H. V. Demir, A. Eychmüller, Angew. Chem. Int. Ed. 50 (2011) 4432.

12. X. Y. Yang, E. Mutlugun, C. Dang, K. Dev, Y. Gao, S. T. Tan, X. W. Sun, H. V. Demir, ACS Nano 8 (2014) 8224.

13. J. Seo, M. J. Cho, D. Lee, A. N. Cartwright, P. N. Prasad, Adv. Mater. 23 (2011) 3984.

14. L. Etgar, T. Moehl, S. Gabriel, S. G. Hickey, A. Eychmüller, M. Grätzel, ACS Nano 6 (2012) 3092.

15. M. F. Oszajca, M. I. Bodnarchuk, M. V. Kovalenko, Chem. Mater. 26 (2014) 5422.

16. S. Kruger, S. G. Hickey, S. Tscharntke, A. Eychmuller, J. Phys. Chem. C 115 (2011) 13047.

17. J. Poppe, S. Gabriel, L. Liebscher, S. G. Hickey, A. Eychmuller, J. Mater. Chem. C 1 (2013) 1515.

18. J. Poppe, S. G. Hickey, A. Eychmuller, J. Phys. Chem. C 118 (2014) 17123.

19. L. M. Peter, Chem. Rev. 90 (1990) 753.

20. P. P. Ingole, S. G. Hickey, C. Waurisch, N. Gaponik, A. Eychmuller, Z. Phys. Chem. Int. 227 (2013) 1173.

21. S. Drouard, S. G. Hickey, D. J. Riley, Chem. Commun. (1999) 67.

22. S. G. Hickey, D. J. Riley, J. Phys. Chem. B 103 (1999) 4599.

23. S. G. Hickey, D. J. Riley, Electrochim. Acta 45 (2000) 3277.

24. S. G. Hickey, D. J. Riley, E. J. Tull, J. Phys. Chem. B 104 (2000) 7623.

25. L. M. Peter, D. J. Riley, E. J. Tull, K. G. U. Wijayantha, Chem. Commun. (2002) 1030.

26. E. Bakkers, J. J. Kelly, D. Vanmaekelbergh, J. Electroanal. Chem. 482 (2000) 48. 
27. E. Bakkers, E. Reitsma, J. J. Kelly, D. Vanmaekelbergh, J. Phys. Chem. B 103 (1999) 2781.

28. E. Bakkers, A. W. Marsman, L. W. Jenneskens, D. Vanmaekelbergh, Angew. Chem. Int. Ed. 39 (2000) 2297.

29. D. Spittel, J. Poppe, C. Meerbach, C. Ziegler, S. G. Hickey, A. Eychmuller, ACS Nano 11 (2017) 12174.

30. D. Yu, C. J. Wang, P. Guyot-Sionnest, Science 300 (2003) 1277.

31. S. Sapra, D. D. Sarma, Phys. Rev. B 69 (2004) 125304. 